\title{
Stacking energies for average B-DNA structures from the combined Density Functional Theory and Symmetry-Adapted Perturbation Theory Approach
}

\author{
- Supporting Information -
}

\author{
Annamaria Fiethen, ${ }^{1}$ Georg Jansen,,${ }^{1, *}$ Andreas Heßelmann, ${ }^{2}$ and Martin Schütz ${ }^{3}$
}

(1) Fachbereich Chemie, Universität Duisburg-Essen, Campus Essen, Universitätsstr. 5, D-45117 Essen, GERMANY

(2) Lehrstuhl für Theoretische Chemie, Universität Erlangen-Nürnberg, Egerlandstr. 3, D91058 Erlangen, GERMANY

(3) Institut für Physikalische und Theoretische Chemie, Universität Regensburg, Universitätsstr. 31, D-93040 Regensburg, GERMANY

$\left(^{*}\right)$ e-mail: georg.jansen@uni-due.de

\section{Further computational details}

Geometries Atomic coordinates generated by the 3DNA program (cf. Ref. 10 of the main text) normally do not contain those of the hydrogen atoms which, in general, are experimentally inaccessible. To obtain them first all atoms belonging to the backbone of DNA were deleted in the atomic coordinate files of the various nucleotides. Then the non-hydrogen atomic positions of the AT and CG dimers were generated with 3DNA using the appropriate complementary base pair parameters (values given in main text). Subsequently the positions of manually added hydrogen atoms were refined in geometry optimizations using the TZVPP basis set (cf. Ref. 12) in frozen-core MP2 calculations. This level of theory is expected to yield reliable $\mathrm{CH}$ and $\mathrm{NH}$ bond lengths and fairly accurate hydrogen bridge geometries, thus also providing a starting point for future investigations of the hydrogen bonds in average B-DNA. The positions of all non-hydrogen atoms were kept fixed during the geometry optimizations. Finally, the optimized hydrogen atom positions were added to the atomic coordinate files of the various nucleotides used in 3DNA and the tetramer structures were generated with the appropriate base pair step parameters (values given in main text). The corresponding structures are shown in Figure S1, full lists of atomic coordinates can be found in Table S6.

Exchange-correlation potential (Cited publications listed as Ref. 18 in the main text.) The LPBE0AC xc potential used in the DFT-SAPT calculations is a localized version of the PBE0AC xc potential. The latter was combined from the PBE xc potential of Perdew, Burke and Ernzerhof in its PBE0 modification by Adamo and Barone and the asymptotically correct LB94 xc potential of van Leeuwen and Baerends through the gradient-regulated seamless connection technique of Grüning et al., taking a fraction of $\frac{3}{4} v_{\mathrm{xc}}$ (LB94) in the long-range part. The remaining $\frac{1}{4}$ of the desired asymptotic $1 / r$ behaviour is already taken into account through the exact exchange part of the PBE0 potential. The shift parameter required for the seamless connection of the asymptotic correction was determined from the ionization 
potentials and HOMO energies given in Table S5. Finally the resulting partially non-local PBE0AC xc potential has been converted to a fully local representation termed LPBE0AC using the methods described by Della Sala and Görling and by Heßelmann and Manby. As discussed in Refs. 4d,f the use of a fully local xc potential is more consistent with the fully local representation of the xc kernel in the adiabatic local density (ALDA) approximation. 
Figures

Figure S1: The ten DNA base tetramer structures investigated (note that steps such as AT-CG and CG-AT have different geometries due to the double-helical structure of DNA).

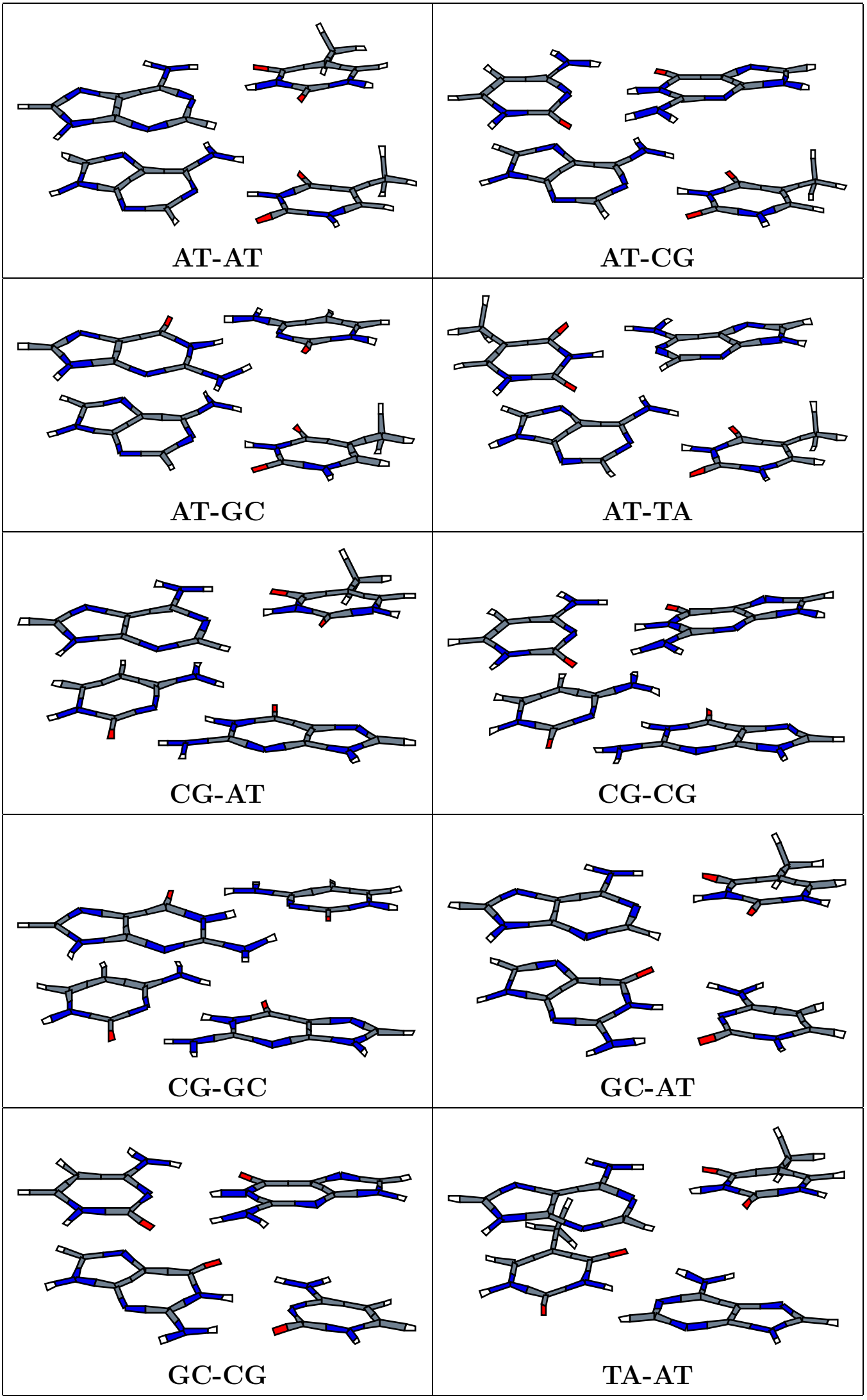




\section{Tables}

All energies given below were obtained with the aug-cc-pVTZ basis set.

TABLE S1: Absolute energies [in $\mathrm{H}$ ] obtained with different methods. Dimer energies were calculated with the full tetramer basis set. Positions of the monomers (denominated as, e.g. a1) and dimers (denominated as, e.g. a1a2) within the tetramer as in the scheme below (dimers approximately perpendicular to the z-axis, "lower" dimer at about $z=0.0 \AA$, "upper" dimer at about $\mathrm{z}=3.32 \AA$; for atomic coordinates see Table S6).

Tetramer (e.g. AT-CG):

$\underline{\mathrm{b} 1} \underline{\mathrm{b} 2}$ hydrogen-bonded dimer b1b2 (e.g. CG)

$\overline{\mathrm{a} 1} \overline{\mathrm{a} 2}$ hydrogen-bonded dimer a1a2 (e.g. AT)

\begin{tabular}{|c|c|c|c|c|c|}
\hline Tetramer & $\mathrm{HF}$ & MP2 & SCS-MP2 & DFT(PBE0) & DFT(LPBE0AC) \\
\hline AT-AT & -1832.73905800 & -1839.76892102 & -1839.56822759 & & \\
\hline b1b2 & -916.37556558 & -919.87140177 & -919.77531763 & -921.11883178 & -921.14433968 \\
\hline a1a2 & -916.37554740 & -919.87130632 & -919.77521468 & 30935 & -921.14431396 \\
\hline AT-CG & -1848.74713456 & -1855.81436095 & -1855.60654317 & & \\
\hline b1b2 & -932.38797297 & -935.91939073 & -935.81632525 & -937.17152361 & -937.19713971 \\
\hline a1a2 & -916.37557153 & -919.87143734 & -919.77535510 & 82006 & 4434344 \\
\hline AT-GC & -1848.74726002 & -1855.81465009 & -1855.60662731 & & \\
\hline b1b2 & -932.38811546 & -935.91948586 & -935.81642640 & -937.17165356 & -937.19728916 \\
\hline $\mathrm{a} 1 \mathrm{a} 2$ & -916.37556908 & -919.87139648 & -919.77531191 & -921.11882299 & -921.14434789 \\
\hline AT-T & 832.72989164 & -1839.76250976 & -1839.56144626 & & \\
\hline b1b2 & -916.37556560 & -919.87151491 & -919.77544003 & 81669 & 4434515 \\
\hline a1a2 & -916.37557342 & -919.87152798 & -919.77545320 & -921.11882378 & 1435050 \\
\hline CG- $f$ & -1848.75192293 & -1855.81516324 & -1855.607 & & \\
\hline b1b2 & -916.37553830 & -919.87121463 & -919.77511658 & 79912 & -921.14432451 \\
\hline a1a2 & -932.38794979 & -935.91914465 & -935.81606054 & -937.17150140 & -937.19711632 \\
\hline CG-CG & -1864.75672064 & -1871.86025581 & -1871.64524971 & & \\
\hline b1b2 & -932.38797591 & -935.91930193 & -935.81622887 & -937. & -937.19716215 \\
\hline a1a2 & -932.38798593 & -935.91936101 & -935.81629201 & -937.17153109 & -937.19714103 \\
\hline CG-GC & -1864.76510050 & -1871.86678233 & -1871.65159951 & & \\
\hline b1b2 & -932.38810862 & -935.91948700 & -935.81642867 & -937.1 & -937.19727317 \\
\hline a1a2 & -932.38797931 & -935.91938763 & -935.81632272 & -937.17152689 & -937.19713648 \\
\hline GC-A & 96448 & -1855.81458033 & -1855.60700515 & & \\
\hline b1b2 & 55629 & -919.87129223 & -919.77519811 & -921. & -921.14434616 \\
\hline a1a2 & -932.38807216 & -935.91922277 & -935.81614211 & -937.17159489 & -937.19721485 \\
\hline GC-C & -1864.7 & -1871.86509046 & -1871.6 & & \\
\hline b1b2 & -932.38797003 & -935.91931250 & -935.81624038 & -937. & -937.19714487 \\
\hline a1a2 & -932.38809790 & -935.91941302 & -935.81634757 & -937.17161448 & -937.19723302 \\
\hline TA-AT & -1832.74044609 & -1839.76576502 & -1839.56580183 & & \\
\hline b1b2 & 2403 & 107516 & -919.77 & -921 & -921.14430804 \\
\hline a1a2 & -916.37551610 & -919.87106879 & -919.77495990 & -921.11877844 & -921.14431095 \\
\hline
\end{tabular}


TABLE S2: Interaction energy contributions obtained at the HF-SAPT level [in $\mathrm{kJ} / \mathrm{mol}]$ used to evaluate $\delta(\mathrm{HF})$.

\begin{tabular}{lrrrrr}
\hline Tetramer & $E_{\text {el }}^{(1)}$ & $E_{\text {exch }}^{(1)}$ & $E_{\text {ind }}^{(2)}$ & $E_{\text {ind-exch }}^{(2)}$ & $\delta(\mathrm{HF})$ \\
\hline AT-AT & -13.65 & 51.48 & -21.21 & 17.38 & -2.35 \\
AT-CG & -8.41 & 60.86 & -28.74 & 22.08 & -2.71 \\
AT-GC & -10.08 & 62.32 & -28.81 & 22.78 & -3.10 \\
AT-TA & -7.34 & 70.41 & -30.18 & 25.96 & -3.07 \\
CG-AT & -27.90 & 67.00 & -32.75 & 27.22 & -3.20 \\
CG-CG & 1.90 & 57.90 & -28.58 & 21.42 & -2.12 \\
CG-GC & -44.13 & 85.04 & -43.09 & 35.26 & -4.24 \\
GC-AT & -3.16 & 48.51 & -22.39 & 17.24 & -1.70 \\
GC-CG & -7.99 & 54.17 & -28.18 & 20.10 & -2.32 \\
TA-AT & -13.99 & 47.46 & -20.61 & 17.31 & -2.35 \\
\hline
\end{tabular}

TABLE S3: Interaction energy contributions obtained with DFT-SAPT using the LPBE0AC xc potential $[$ in $\mathrm{kJ} / \mathrm{mol}]$.

\begin{tabular}{lrrrrrr}
\hline Tetramer & $E_{\text {el }}^{(1)}$ & $E_{\text {exch }}^{(1)}$ & $E_{\text {ind }}^{(2)}$ & $E_{\text {ind-exch }}^{(2)}$ & $E_{\text {disp }}^{(2)}$ & $E_{\text {disp-exch }}^{(2)}$ \\
\hline AT-AT & -15.87 & 57.73 & -24.89 & 21.75 & -94.25 & 10.21 \\
AT-CG & -12.73 & 67.48 & -33.02 & 27.48 & -99.19 & 11.62 \\
AT-GC & -14.47 & 70.06 & -34.36 & 29.39 & -101.52 & 12.04 \\
AT-TA & -11.68 & 77.12 & -35.89 & 32.68 & -102.35 & 12.52 \\
CG-AT & -28.19 & 73.99 & -38.94 & 34.44 & -97.86 & 12.41 \\
CG-CG & -4.81 & 65.92 & -34.16 & 28.10 & -100.60 & 11.81 \\
CG-GC & -43.50 & 94.02 & -52.72 & 46.37 & -110.47 & 15.54 \\
GC-AT & -8.00 & 54.73 & -26.00 & 21.60 & -92.32 & 9.98 \\
GC-CG & -12.94 & 60.53 & -31.67 & 24.77 & -96.80 & 10.93 \\
TA-AT & -15.81 & 54.02 & -24.58 & 21.96 & -86.76 & 9.43 \\
\hline
\end{tabular}


TABLE S4: Interaction energies from HF, MP2, SCS-MP2 and DFT-SAPT(LPBE0AC) [in $\mathrm{kJ} / \mathrm{mol}$ ] (all HF/MP2/SCS-MP2-energies are counterpoise-corrected).

\begin{tabular}{lrrrr}
\hline Tetramer & HF & MP2 & SCS-MP2 & DFT-SAPT \\
\hline AT-AT & 31.65 & -68.82 & -46.46 & -47.66 \\
AT-CG & 43.08 & -61.79 & -39.02 & -41.07 \\
AT-GC & 43.12 & -62.40 & -39.09 & -41.96 \\
AT-TA & 55.78 & -51.11 & -27.71 & -30.66 \\
CG-AT & 30.36 & -65.12 & -43.23 & -47.36 \\
CG-CG & 50.52 & -56.69 & -33.42 & -35.86 \\
CG-GC & 28.85 & -73.27 & -49.49 & -54.99 \\
GC-AT & 38.50 & -63.18 & -41.13 & -41.72 \\
GC-CG & 35.77 & -69.22 & -46.67 & -47.51 \\
TA-AT & 27.81 & -62.02 & -41.68 & -44.08 \\
\hline
\end{tabular}

TABLE S5: Ionization potentials (IP) [in H] (obtained from DFT(PBE0)/aug-cc-pVTZ calculations on the neutral and ionized dimers) and HOMO-Energies $\left(\epsilon_{\mathrm{HOMO}}\right)[$ in $\mathrm{H}]$ (from DFT(PBE0)/aug-cc-pVTZ for the neutral dimers) used for the asymptotic shift term employed in the LPBE0AC xc potential.

\begin{tabular}{lrrr}
\hline Dimer & IP & $\epsilon_{\text {HOMO }}$ & shift \\
\hline AT & 0.2917 & -0.2349 & 0.0568 \\
CG & 0.2628 & -0.2043 & 0.0585 \\
\hline
\end{tabular}


TABLE S6: Atomic coordinates [in $\AA$ ] for the tetramers (note that steps such as AT-CG and CG-AT have different geometries due to the double-helical structure of DNA).

\begin{tabular}{|c|c|c|c|}
\hline \multicolumn{4}{|c|}{ Tetramer 1 - AT-AT } \\
\hline $\mathrm{H}$ & -2.131063366 & 4.990277619 & $-0.145534 \mathrm{~S}$ \\
\hline $\mathrm{N}$ & -1.309008019 & 4.416421066 & -0.065994647 \\
\hline $\mathrm{C}$ & -0.002694710 & 4.822012314 & 0.066169185 \\
\hline $\mathrm{N}$ & 0.851474892 & 3.831315542 & 0.147090114 \\
\hline $\mathrm{C}$ & 0.055609867 & 2.696295159 & 0.062682822 \\
\hline $\mathrm{C}$ & 0.359583204 & 1.324821159 & 0.087019545 \\
\hline $\mathrm{N}$ & 1.598077651 & 0.842065626 & 0.208525105 \\
\hline $\mathrm{N}$ & -0.667574384 & 0.453635522 & -0.019318945 \\
\hline $\mathrm{C}$ & -1.908069766 & 0.938380973 & -0.141015514 \\
\hline $\mathrm{N}$ & -2.320922337 & 2.203305218 & -0.176686352 \\
\hline $\mathrm{C}$ & -1.277670434 & 3.042571866 & -0.068881161 \\
\hline $\mathrm{H}$ & 0.262301524 & 5.865354486 & 0.097187041 \\
\hline $\mathrm{H}$ & 1.769522246 & -0.151500443 & 0.071546212 \\
\hline $\mathrm{H}$ & 2.364926308 & 1.490291745 & 0.195447040 \\
\hline $\mathrm{H}$ & -2.678914159 & 0.180457949 & -0.213234712 \\
\hline $\mathrm{H}$ & -4.732964538 & 3.004663028 & 3.204568689 \\
\hline $\mathrm{N}$ & -3.729899019 & 3.023961410 & 3.274569672 \\
\hline $\mathrm{C}$ & -2.910286518 & 4.120538364 & 3.393650867 \\
\hline $\mathrm{N}$ & -1.636219165 & 3.821486535 & 3.464038249 \\
\hline $\mathrm{C}$ & -1.613703435 & 2.435040863 & 3.386202281 \\
\hline $\mathrm{C}$ & -0.561447610 & 1.504272179 & 3.405184789 \\
\hline $\mathrm{N}$ & 0.725351774 & 1.842244698 & 3.512920206 \\
\hline $\mathrm{N}$ & -0.881322861 & 0.195222532 & 3.308262927 \\
\hline $\mathrm{C}$ & -2.170912367 & -0.142317106 & 3.200360654 \\
\hline $\mathrm{N}$ & -3.248727141 & 0.638200305 & 3.171019816 \\
\hline $\mathrm{C}$ & -2.897057021 & 1.930893592 & 3.269189022 \\
\hline $\mathrm{H}$ & -3.308874376 & 5.120529864 & 3.423529284 \\
\hline $\mathrm{H}$ & 1.446750664 & 1.138526602 & 3.372583166 \\
\hline $\mathrm{H}$ & 0.964571315 & 2.817331740 & 3.492813714 \\
\hline $\mathrm{H}$ & -2.349690413 & -1.208917364 & 3.135043748 \\
\hline $\mathrm{H}$ & 1.128520864 & -5.057994322 & 3.526271130 \\
\hline $\mathrm{N}$ & 1.471520484 & -4.117112320 & 3.430975165 \\
\hline $\mathrm{C}$ & 0.530987859 & -3.112083159 & 3.445933998 \\
\hline $\mathrm{O}$ & -0.664608919 & -3.322148874 & 3.564886675 \\
\hline $\mathrm{N}$ & 1.045615710 & -1.845951068 & 3.315750653 \\
\hline $\mathrm{C}$ & 2.374600620 & -1.494494488 & 3.175602943 \\
\hline $\mathrm{O}$ & 2.687293341 & -0.311875778 & 3.068737376 \\
\hline $\mathrm{C}$ & 3.306222775 & -2.599505571 & 3.168142034 \\
\hline $\mathrm{C}$ & 4.767653400 & -2.316663570 & 3.019983565 \\
\hline $\mathrm{C}$ & 2.813786719 & -3.838612203 & 3.294563886 \\
\hline $\mathrm{H}$ & 0.333241109 & -1.079714749 & 3.309640681 \\
\hline $\mathrm{H}$ & 5.342116367 & -3.239924428 & 3.031632064 \\
\hline $\mathrm{H}$ & 4.96 & -1.790621716 & 2.08731 \\
\hline
\end{tabular}




$\begin{array}{rrrr}\mathrm{H} & 5.116336808 & -1.673423231 & 3.826518591 \\ \mathrm{H} & 3.461793964 & -4.704110251 & 3.295882965 \\ \mathrm{H} & -2.131593200 & -4.977324439 & 0.191890681 \\ \mathrm{~N} & -1.300104784 & -4.417889046 & 0.104437519 \\ \mathrm{C} & -1.470406710 & -3.051951696 & 0.115456215 \\ \mathrm{O} & -2.562322665 & -2.518966482 & 0.222111307 \\ \mathrm{~N} & -0.308541510 & -2.330316453 & -0.003673265 \\ \mathrm{C} & 0.974607464 & -2.827349500 & -0.129573464 \\ \mathrm{O} & 1.923783107 & -2.054548581 & -0.227697562 \\ \mathrm{C} & 1.078857062 & -4.268925116 & -0.133668089 \\ \mathrm{C} & 2.428905548 & -4.899330068 & -0.266666892 \\ \mathrm{C} & -0.049131734 & -4.981745973 & -0.017958533 \\ \mathrm{H} & -0.434402580 & -1.291706926 & -0.012742044 \\ \mathrm{H} & 2.350849763 & -5.983905591 & -0.254127781 \\ \mathrm{H} & 2.904788186 & -4.589224753 & -1.194880449 \\ \mathrm{H} & 3.080582289 & -4.582612432 & 0.546256453 \\ \mathrm{H} & -0.033633497 & -6.062834154 & -0.014772681\end{array}$

Tetramer 2 - AT-CG

$\begin{array}{lrrr}\mathrm{H} & -2.131063366 & 4.990277619 & -0.145534971 \\ \mathrm{~N} & -1.309008019 & 4.416421066 & -0.065994647 \\ \mathrm{C} & -0.002694710 & 4.822012314 & 0.066169185 \\ \mathrm{~N} & 0.851474892 & 3.831315542 & 0.147090114 \\ \mathrm{C} & 0.055609867 & 2.696295159 & 0.062682822 \\ \mathrm{C} & 0.359583204 & 1.324821159 & 0.087019545 \\ \mathrm{~N} & 1.598077651 & 0.842065626 & 0.208525105 \\ \mathrm{~N} & -0.667574384 & 0.453635522 & -0.019318945 \\ \mathrm{C} & -1.908069766 & 0.938380973 & -0.141015514 \\ \mathrm{~N} & -2.320922337 & 2.203305218 & -0.176686352 \\ \mathrm{C} & -1.277670434 & 3.042571866 & -0.068881161 \\ \mathrm{H} & 0.262301524 & 5.865354486 & 0.097187041 \\ \mathrm{H} & 1.769522246 & -0.151500443 & 0.071546212 \\ \mathrm{H} & 2.364926308 & 1.490291745 & 0.195447040 \\ \mathrm{H} & -2.678914159 & 0.180457949 & -0.213234712 \\ \mathrm{H} & -4.755254235 & 3.036679869 & 3.204870870 \\ \mathrm{~N} & -3.751134604 & 3.062954369 & 3.275343681 \\ \mathrm{C} & -3.081792054 & 1.837466600 & 3.251151143 \\ \mathrm{O} & -3.744199337 & 0.794254282 & 3.146927104 \\ \mathrm{~N} & -1.731827585 & 1.818651658 & 3.342796825 \\ \mathrm{C} & -1.056085852 & 2.964680546 & 3.454827613 \\ \mathrm{~N} & 0.275270935 & 2.898707271 & 3.543499106 \\ \mathrm{C} & -1.713353012 & 4.227562984 & 3.481990628 \\ \mathrm{C} & -3.049277101 & 4.230634237 & 3.390407893 \\ \mathrm{H} & -3.633578991 & 5.138981495 & 3.399271291 \\ \mathrm{H} & -1.164585317 & 5.151392368 & 3.570569886 \\ \mathrm{H} & 0.809270014 & 3.747531929 & 3.576569995 \\ \mathrm{H} & 0.774704635 & 2.004474466 & 3.420179723\end{array}$




$\begin{array}{lrrr}\mathrm{H} & 1.160901374 & -5.104920031 & 3.525577996 \\ \mathrm{~N} & 1.497256623 & -4.161419579 & 3.431694851 \\ \mathrm{C} & 2.796268240 & -3.734222974 & 3.289087422 \\ \mathrm{~N} & 2.900305987 & -2.436777864 & 3.192804848 \\ \mathrm{C} & 1.592168613 & -1.977824609 & 3.276682855 \\ \mathrm{C} & 1.075603479 & -0.658347481 & 3.233340380 \\ \mathrm{O} & 1.692966336 & 0.407009965 & 3.10763587 \\ \mathrm{~N} & -0.309827620 & -0.645253630 & 3.353948092 \\ \mathrm{C} & -1.098054311 & -1.760719564 & 3.498001667 \\ \mathrm{~N} & -2.418247058 & -1.541911239 & 3.600075305 \\ \mathrm{~N} & -0.629545994 & -2.996520419 & 3.538930929 \\ \mathrm{C} & 0.714529965 & -3.031933606 & 3.424455064 \\ \mathrm{H} & -0.788179579 & 0.266947243 & 3.316505196 \\ \mathrm{H} & 3.619552272 & -4.428239604 & 3.262510784 \\ \mathrm{H} & -2.850591255 & -0.649193943 & 3.360702530 \\ \mathrm{H} & -2.984882921 & -2.370629919 & 3.581686530 \\ \mathrm{H} & -2.131593200 & -4.977324439 & 0.191890681 \\ \mathrm{~N} & -1.300104784 & -4.417889046 & 0.104437519 \\ \mathrm{C} & -1.470406710 & -3.051951696 & 0.115456215 \\ \mathrm{O} & -2.562322665 & -2.518966482 & 0.222111307 \\ \mathrm{~N} & -0.308541510 & -2.330316453 & -0.003673265 \\ \mathrm{C} & 0.974607464 & -2.827349500 & -0.129573464 \\ \mathrm{O} & 1.923783107 & -2.054548581 & -0.227697562 \\ \mathrm{C} & 1.078857062 & -4.268925116 & -0.133668089 \\ \mathrm{C} & 2.428905548 & -4.899330068 & -0.266666892 \\ \mathrm{C} & -0.049131734 & -4.981745973 & -0.017958533 \\ \mathrm{H} & -0.434402580 & -1.291706926 & -0.012742044 \\ \mathrm{H} & 2.350849763 & -5.983905591 & -0.254127781 \\ \mathrm{H} & 2.904788186 & -4.589224753 & -1.194880449 \\ \mathrm{H} & 3.080582289 & -4.582612432 & 0.546256453 \\ \mathrm{H} & -0.033633497 & -6.062834154 & -0.014772681\end{array}$

Tetramer 3 - AT-GC

$\begin{array}{lrrr}\mathrm{H} & -2.131063366 & 4.990277619 & -0.145534971 \\ \mathrm{~N} & -1.309008019 & 4.416421066 & -0.065994647 \\ \mathrm{C} & -0.002694710 & 4.822012314 & 0.066169185 \\ \mathrm{~N} & 0.851474892 & 3.831315542 & 0.147090114 \\ \mathrm{C} & 0.055609867 & 2.696295159 & 0.062682822 \\ \mathrm{C} & 0.359583204 & 1.324821159 & 0.087019545 \\ \mathrm{~N} & 1.598077651 & 0.842065626 & 0.208525105 \\ \mathrm{~N} & -0.667574384 & 0.453635522 & -0.019318945 \\ \mathrm{C} & -1.908069766 & 0.938380973 & -0.141015514 \\ \mathrm{~N} & -2.320922337 & 2.203305218 & -0.176686352 \\ \mathrm{C} & -1.277670434 & 3.042571866 & -0.068881161 \\ \mathrm{H} & 0.262301524 & 5.865354486 & 0.097187041 \\ \mathrm{H} & 1.769522246 & -0.151500443 & 0.071546212 \\ \mathrm{H} & 2.364926308 & 1.490291745 & 0.195447040\end{array}$




\begin{tabular}{|c|c|c|c|}
\hline & & & \\
\hline & & & \\
\hline & & & \\
\hline & -2.94 & & \\
\hline & & & \\
\hline & -1.6 & & \\
\hline & & & \\
\hline & & & \\
\hline & -0. & & \\
\hline & -2.2 & & \\
\hline & & & \\
\hline & -3.31 & & \\
\hline & -2.9 & & \\
\hline & -0.2 & & \\
\hline & -3. & & \\
\hline & -1.7 & & \\
\hline & -3.4 & & \\
\hline & 30 & & \\
\hline & & & \\
\hline & & & \\
\hline & -0.6 & & \\
\hline & & & \\
\hline & & & \\
\hline & & & \\
\hline & 3.2 & & \\
\hline & & & \\
\hline & 3.5 & & \\
\hline & & & \\
\hline & & & \\
\hline & & & \\
\hline & -2.1 & -4 & \\
\hline & -1.30 & & \\
\hline & & & \\
\hline & -2.5 & & 30 \\
\hline & -0.3 & -2. & -0.0 \\
\hline & & -2.8 & -0.1 \\
\hline & & & $\pi$ \\
\hline & 1.07 & & \\
\hline & 2.42 & & \\
\hline & -0.04 & & -0. \\
\hline & -0.4 & & -0.0 \\
\hline & & & \\
\hline & 88186 & -4.589224753 & -1.1 ? \\
\hline & 3.080582289 & -4.582612432 & 0.546256 \\
\hline & 022622407 & -6.062834154 & 0014770 \\
\hline
\end{tabular}

Tetramer 4 - AT-TA 


$\begin{array}{rrrr}\mathrm{H} & -2.131063366 & 4.990277619 & -0.145534971 \\ \mathrm{~N} & -1.309008019 & 4.416421066 & -0.065994647 \\ \mathrm{C} & -0.002694710 & 4.822012314 & 0.066169185 \\ \mathrm{~N} & 0.851474892 & 3.831315542 & 0.147090114 \\ \mathrm{C} & 0.055609867 & 2.696295159 & 0.062682822 \\ \mathrm{C} & 0.359583204 & 1.324821159 & 0.087019545 \\ \mathrm{~N} & 1.598077651 & 0.842065626 & 0.208525105 \\ \mathrm{~N} & -0.667574384 & 0.453635522 & -0.019318945 \\ \mathrm{C} & -1.908069766 & 0.938380973 & -0.141015514 \\ \mathrm{~N} & -2.320922337 & 2.203305218 & -0.176686352 \\ \mathrm{C} & -1.277670434 & 3.042571866 & -0.068881161 \\ \mathrm{H} & 0.262301524 & 5.865354486 & 0.097187041 \\ \mathrm{H} & 1.769522246 & -0.151500443 & 0.071546212 \\ \mathrm{H} & 2.364926308 & 1.490291745 & 0.195447040 \\ \mathrm{H} & -2.678914159 & 0.180457949 & -0.213234712 \\ \mathrm{H} & -4.728131110 & 2.993338926 & 3.202244322 \\ \mathrm{~N} & -3.725462667 & 3.029698815 & 3.275202887 \\ \mathrm{C} & -3.060143904 & 1.824826380 & 3.251914326 \\ \mathrm{O} & -3.631332780 & 0.751822122 & 3.151245907 \\ \mathrm{~N} & -1.694457526 & 1.924232496 & 3.351408753 \\ \mathrm{C} & -0.947195500 & 3.080584159 & 3.468942987 \\ \mathrm{O} & 0.276267363 & 3.013568868 & 3.549140083 \\ \mathrm{C} & -1.710509434 & 4.307799983 & 3.486779998 \\ \mathrm{C} & -0.987467621 & 5.611348907 & 3.612082587 \\ \mathrm{C} & -3.043566734 & 4.221180693 & 3.390258839 \\ \mathrm{H} & -1.185383477 & 1.010188448 & 3.351107979 \\ \mathrm{H} & -1.688531379 & 6.442651078 & 3.611526809 \\ \mathrm{H} & -0.406767913 & 5.638417036 & 4.532009304 \\ \mathrm{H} & -2.134069528 & -1.497854086 & 3.563141599 \\ \mathrm{H} & -2.286032258 & 5.740201623 & 2.789147348 \\ \mathrm{H} & -3.666785523 & 5.104662347 & 3.398037474 \\ \mathrm{H} & 1.137773469 & -5.066130964 & 3.523994214 \\ \mathrm{~N} & 1.464702353 & -4.119548136 & 3.431736675 \\ \mathrm{C} & 2.759130411 & -3.680868740 & 3.288759785 \\ \mathrm{~N} & 2.866843981 & -2.378320114 & 3.191812038 \\ \mathrm{C} & 1.555974867 & -1.927486060 & 3.276475019 \\ \mathrm{C} & 0.995012237 & -0.639906100 & 3.239168508 \\ \mathrm{~N} & 1.712124863 & 0.477414987 & 3.101226242 \\ \mathrm{~N} & -0.347627289 & -0.538196860 & 3.350070869 \\ \mathrm{C} & -1.065186830 & -1.658301669 & 3.488239329 \\ \mathrm{~N} & -0.654898823 & -2.923613285 & 3.537262436 \\ \mathrm{C} & 0.681975695 & -2.990062162 & 3.424496887 \\ \mathrm{H} & 3.586984653 & -4.369135135 & 3.262402183 \\ & -26958851 & 1.383368677 & 3.229308769 \\ \end{array}$




\begin{tabular}{|c|c|c|c|}
\hline $\mathrm{J}$ & -1.300104784 & -4.417889046 & 0.104437519 \\
\hline C & -1.470406710 & -3.051951696 & 0.115456215 \\
\hline $\mathrm{O}$ & -2.562322665 & -2.518966482 & 0.222111307 \\
\hline N & -0.308541510 & -2.330316453 & -0.003673265 \\
\hline $\mathrm{C}$ & 0.974607464 & -2.827349500 & -0.129573464 \\
\hline & 1.923783107 & -2.054548581 & -0.227697562 \\
\hline & 1.078857062 & -4.268925116 & -0.133668089 \\
\hline $\mathrm{c}$ & 2.428905548 & -4.899330068 & -0.2666668892 \\
\hline $\mathrm{C}$ & -0.049131734 & -4.981745973 & -0.017958533 \\
\hline & -0.434402580 & -1.291706926 & -0.012742044 \\
\hline & 2.350849763 & -5.983905591 & -0.254127781 \\
\hline ] & 2.904788186 & -4.589224753 & -1.194880449 \\
\hline & 3.080582289 & -4.582612432 & 0.546256453 \\
\hline & -0.033633497 & -6.062834154 & -0.014772681 \\
\hline \multicolumn{4}{|c|}{ Tetramer 5 - CG-AT } \\
\hline $\mathrm{I}$ & -2.130279976 & 5.029281776 & -0.145286025 \\
\hline & -1.303276559 & 4.460450209 & -0.065229817 \\
\hline $\mathrm{C}$ & -1.481838314 & 3.075542209 & -0.089115617 \\
\hline ) & -2.629768109 & 2.620745739 & -0.204681004 \\
\hline N & -0.401781684 & 2.266985785 & 0.015149353 \\
\hline $\mathrm{C}$ & 0.817284709 & 2.797137614 & 0.139150031 \\
\hline $\mathrm{s}$ & 1.854601792 & 1.961361611 & 0.240034817 \\
\hline 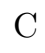 & 1.027562950 & 4.205205716 & 0.166302969 \\
\hline c & -0.050389633 & 4.992772642 & 0.062156124 \\
\hline $\mathrm{H}$ & 0.010725825 & 6.071098181 & 0.069962057 \\
\hline $\mathrm{H}$ & 2.013557794 & 4.630189076 & 0.264569501 \\
\hline $\mathrm{H}$ & 2.785147320 & 2.334257763 & 0.282289779 \\
\hline $\mathrm{H}$ & 1.734328253 & 0.944158208 & 0.117048998 \\
\hline $\mathrm{H}$ & -4.732964538 & 3.004663028 & 3.204568689 \\
\hline N & -3.729899019 & 3.023961410 & 3.274569672 \\
\hline $\mathrm{C}$ & -2.910286518 & 4.120538364 & 3.393650867 \\
\hline $\mathrm{N}$ & -1.636219165 & 3.821486535 & 3.464038249 \\
\hline $\mathrm{C}$ & -1.613703435 & 2.435040863 & 3.386202281 \\
\hline C & -0.561447610 & 1.504272179 & 3.405184789 \\
\hline N & 0.725351774 & 1.842244698 & 3.512920206 \\
\hline N & -0.881322861 & 0.195222532 & 3.308262927 \\
\hline & -2.170912367 & -0.142317106 & 3.200360654 \\
\hline N & -3.248727141 & 0.638200305 & 3.171019816 \\
\hline C & -2.897057021 & 1.930893592 & 3.269189022 \\
\hline $\mathrm{I}$ & -3.308874376 & 5.120529864 & 3.423529284 \\
\hline & 1.446750664 & 1.138526602 & 3.372583166 \\
\hline $\mathrm{H}$ & 0.964571315 & 2.817331740 & 3.492813714 \\
\hline $\mathrm{H}$ & -2.349690413 & -1.208917364 & 3.135043748 \\
\hline 1 & 1.128520864 & -5.057994322 & 3.526271130 \\
\hline & 1.471520484 & -4.117112320 & 3.430975165 \\
\hline & 0.530987859 & -3.112083159 & 3.445933998 \\
\hline
\end{tabular}




$\begin{array}{rrrr}\mathrm{O} & -0.664608919 & -3.322148874 & 3.564886675 \\ \mathrm{~N} & 1.045615710 & -1.845951068 & 3.315750653 \\ \mathrm{C} & 2.374600620 & -1.494494488 & 3.175602943 \\ \mathrm{O} & 2.687293341 & -0.311875778 & 3.068737376 \\ \mathrm{C} & 3.306222775 & -2.599505571 & 3.168142034 \\ \mathrm{C} & 4.767653400 & -2.316663570 & 3.019983565 \\ \mathrm{C} & 2.813786719 & -3.838612203 & 3.294563886 \\ \mathrm{H} & 0.333241109 & -1.079714749 & 3.309640681 \\ \mathrm{H} & 5.342116367 & -3.239924428 & 3.031632064 \\ \mathrm{H} & 4.961610277 & -1.790621716 & 2.087314975 \\ \mathrm{H} & 5.116336808 & -1.673423231 & 3.826518591 \\ \mathrm{H} & 3.461793964 & -4.704110251 & 3.295882965 \\ \mathrm{H} & -2.132972176 & -5.034321954 & 0.191272867 \\ \mathrm{~N} & -1.305334654 & -4.468860540 & 0.105182676 \\ \mathrm{C} & -0.001890354 & -4.887004567 & -0.023088638 \\ \mathrm{~N} & 0.845869394 & -3.898647248 & -0.112033684 \\ \mathrm{C} & 0.056502273 & -2.758318150 & -0.038236004 \\ \mathrm{C} & 0.414608166 & -1.387276418 & -0.079982102 \\ \mathrm{O} & 1.541528413 & -0.888450349 & -0.194648176 \\ \mathrm{~N} & -0.699730804 & -0.562167449 & 0.027632258 \\ \mathrm{C} & -1.994518731 & -1.001073436 & 0.158791542 \\ \mathrm{~N} & -2.934976761 & -0.047912035 & 0.249494557 \\ \mathrm{~N} & -2.342294163 & -2.276176391 & 0.198081537 \\ \mathrm{C} & -1.274592939 & -3.095024869 & 0.096099844 \\ \mathrm{H} & -0.550154140 & 0.456927742 & -0.009848586 \\ \mathrm{H} & 0.256483822 & -5.932428481 & -0.045306293 \\ \mathrm{H} & -2.757512993 & 0.928059935 & 0.010433216 \\ \mathrm{H} & -3.880261048 & -0.385335714 & 0.221711881\end{array}$

Tetramer 6 - CG-CG

$\begin{array}{lrrr}\mathrm{H} & -2.130279976 & 5.029281776 & -0.145286025 \\ \mathrm{~N} & -1.303276559 & 4.460450209 & -0.065229817 \\ \mathrm{C} & -1.481838314 & 3.075542209 & -0.089115617 \\ \mathrm{O} & -2.629768109 & 2.620745739 & -0.204681004 \\ \mathrm{~N} & -0.401781684 & 2.266985785 & 0.015149353 \\ \mathrm{C} & 0.817284709 & 2.797137614 & 0.139150031 \\ \mathrm{~N} & 1.854601792 & 1.961361611 & 0.240034817 \\ \mathrm{C} & 1.027562950 & 4.205205716 & 0.166302969 \\ \mathrm{C} & -0.050389633 & 4.992772642 & 0.062156124 \\ \mathrm{H} & 0.010725825 & 6.071098181 & 0.069962057 \\ \mathrm{H} & 2.013557794 & 4.630189076 & 0.264569501 \\ \mathrm{H} & 2.785147320 & 2.334257763 & 0.282289779 \\ \mathrm{H} & 1.734328253 & 0.944158208 & 0.117048998 \\ \mathrm{H} & -4.755254235 & 3.036679869 & 3.204870870 \\ \mathrm{~N} & -3.751134604 & 3.062954369 & 3.275343681 \\ \mathrm{C} & -3.081792054 & 1.837466600 & 3.251151143 \\ \mathrm{O} & -3.744199337 & 0.794254282 & 3.146927104\end{array}$




$\begin{array}{lrrr}\mathrm{N} & -1.731827585 & 1.818651658 & 3.342796825 \\ \mathrm{C} & -1.056085852 & 2.964680546 & 3.454827613 \\ \mathrm{~N} & 0.275270935 & 2.898707271 & 3.543499106 \\ \mathrm{C} & -1.713353012 & 4.227562984 & 3.481990628 \\ \mathrm{C} & -3.049277101 & 4.230634237 & 3.390407893 \\ \mathrm{H} & -3.633578991 & 5.138981495 & 3.399271291 \\ \mathrm{H} & -1.164585317 & 5.151392368 & 3.570569886 \\ \mathrm{H} & 0.809270014 & 3.747531929 & 3.576569995 \\ \mathrm{H} & 0.774704635 & 2.004474466 & 3.420179723 \\ \mathrm{H} & 1.160901374 & -5.104920031 & 3.525577996 \\ \mathrm{~N} & 1.497256623 & -4.161419579 & 3.431694851 \\ \mathrm{C} & 2.796268240 & -3.734222974 & 3.289087422 \\ \mathrm{~N} & 2.900305987 & -2.436777864 & 3.192804848 \\ \mathrm{C} & 1.592168613 & -1.977824609 & 3.276682855 \\ \mathrm{C} & 1.075603479 & -0.658347481 & 3.233340380 \\ \mathrm{O} & 1.692966336 & 0.407009965 & 3.107635897 \\ \mathrm{~N} & -0.309827620 & -0.645253630 & 3.353948092 \\ \mathrm{C} & -1.098054311 & -1.760719564 & 3.498001667 \\ \mathrm{~N} & -2.418247058 & -1.541911239 & 3.600075305 \\ \mathrm{~N} & -0.629545994 & -2.996520419 & 3.538930929 \\ \mathrm{C} & 0.714529965 & -3.031933606 & 3.424455064 \\ \mathrm{H} & -0.788179579 & 0.266947243 & 3.316505196 \\ \mathrm{H} & 3.619552272 & -4.428239604 & 3.262510784 \\ \mathrm{H} & -2.850591255 & -0.649193943 & 3.360702530 \\ \mathrm{H} & -2.984882921 & -2.370629919 & 3.581686530 \\ \mathrm{H} & -2.132972176 & -5.034321954 & 0.191272867 \\ \mathrm{~N} & -1.305334654 & -4.468860540 & 0.105182676 \\ \mathrm{C} & -0.001890354 & -4.887004567 & -0.023088638 \\ \mathrm{~N} & 0.845869394 & -3.898647248 & -0.112033684 \\ \mathrm{C} & 0.056502273 & -2.758318150 & -0.038236004 \\ \mathrm{C} & 0.414608166 & -1.387276418 & -0.079982102 \\ \mathrm{O} & 1.541528413 & -0.888450349 & -0.194648176 \\ \mathrm{~N} & -0.699730804 & -0.562167449 & 0.027632258 \\ \mathrm{C} & -1.994518731 & -1.001073436 & 0.158791542 \\ \mathrm{~N} & -2.934976761 & -0.047912035 & 0.249494557 \\ \mathrm{~N} & -2.342294163 & -2.276176391 & 0.198081537 \\ \mathrm{C} & -1.274592939 & -3.095024869 & 0.096099844 \\ \mathrm{H} & -0.550154140 & 0.456927742 & -0.009848586 \\ \mathrm{H} & 0.256483822 & -5.932428481 & -0.045306293 \\ \mathrm{H} & -2.757512993 & 0.928059935 & 0.010433216 \\ \mathrm{H} & -3.880261048 & -0.385335714 & 0.221711881\end{array}$

Tetramer 7 - CG-GC

$\begin{array}{llll}\mathrm{H} & -2.130279976 & 5.029281776 & -0.145286025 \\ \mathrm{~N} & -1.303276559 & 4.460450209 & -0.065229817 \\ \mathrm{C} & -1.481838314 & 3.075542209 & -0.089115617 \\ \mathrm{O} & -2.629768109 & 2.620745739 & -0.204681004\end{array}$




\begin{tabular}{|c|c|c|c|}
\hline & & & \\
\hline & 0.817284709 & & \\
\hline & & & \\
\hline & 0 & & \\
\hline & & & \\
\hline & & & \\
\hline & & & \\
\hline & 17320 & & \\
\hline & 1.7 & & \\
\hline & 43 & & \\
\hline & & 91 & \\
\hline & -2.9 & 55 & \\
\hline & & & \\
\hline & -1.6 & & \\
\hline & & 94 & \\
\hline & & & \\
\hline & -0.9 & 3 & \\
\hline & -2.2 & 1 & \\
\hline & -2.4 & & \\
\hline & -3.3 & & \\
\hline & -2. & & \\
\hline & -0.2 & .47 & \\
\hline & -3.3 & & \\
\hline & -1.7 & & \\
\hline & -3.4 & & \\
\hline & & 37 & \\
\hline & & & \\
\hline & & & \\
\hline & -0.6 & & \\
\hline & & 20 & \\
\hline & & & \\
\hline & & 50 & \\
\hline & 3.2 & -2.5 & \\
\hline & & -3.8 & \\
\hline & & & \\
\hline & & & \\
\hline & 3.54 & -0.03 & \\
\hline & & & \\
\hline & -2.132972176 & -5.03 & 86 \\
\hline & -1.3 & -4.4 & \\
\hline & -0.00 & -4.88 & \\
\hline & & -3.89 & -0 \\
\hline & 2273 & -2.75 & -0 . \\
\hline & & -1.3 & \\
\hline & & -0.8 & -0.1 \\
\hline & 0.699730804 & -0.562167449 & 0.027632 \\
\hline
\end{tabular}




$\begin{array}{lrrr}\mathrm{C} & -1.994518731 & -1.001073436 & 0.158791542 \\ \mathrm{~N} & -2.934976761 & -0.047912035 & 0.249494557 \\ \mathrm{~N} & -2.342294163 & -2.276176391 & 0.198081537 \\ \mathrm{C} & -1.274592939 & -3.095024869 & 0.096099844 \\ \mathrm{H} & -0.550154140 & 0.456927742 & -0.009848586 \\ \mathrm{H} & 0.256483822 & -5.932428481 & -0.045306293 \\ \mathrm{H} & -2.757512993 & 0.928059935 & 0.010433216 \\ \mathrm{H} & -3.880261048 & -0.385335714 & 0.221711881\end{array}$

Tetramer 8 - GC-AT

$\begin{array}{lrrr}\mathrm{H} & -2.135188422 & 5.035260905 & -0.146754760 \\ \mathrm{~N} & -1.307305565 & 4.469429934 & -0.065592656 \\ \mathrm{C} & -0.004042501 & 4.887005860 & 0.066319186 \\ \mathrm{~N} & 0.844146002 & 3.898278929 & 0.146651742 \\ \mathrm{C} & 0.055273397 & 2.758293790 & 0.062920655 \\ \mathrm{C} & 0.413973979 & 1.387095838 & 0.092721127 \\ \mathrm{O} & 1.541110686 & 0.887778524 & 0.203039132 \\ \mathrm{~N} & -0.700007225 & 0.562472505 & -0.022079726 \\ \mathrm{C} & -1.994985640 & 1.001942901 & -0.149412665 \\ \mathrm{~N} & -2.935030350 & 0.049200089 & -0.248418098 \\ \mathrm{~N} & -2.343314147 & 2.277188808 & -0.177592940 \\ \mathrm{C} & -1.275967980 & 3.095580734 & -0.068479170 \\ \mathrm{H} & -0.549988506 & -0.456845579 & 0.006521469 \\ \mathrm{H} & 0.253878274 & 5.932308498 & 0.097644373 \\ \mathrm{H} & -2.757142852 & -0.928740518 & -0.017868306 \\ \mathrm{H} & -3.880460843 & 0.385958898 & -0.217698577 \\ \mathrm{H} & -4.732964538 & 3.004663028 & 3.204568689 \\ \mathrm{~N} & -3.729899019 & 3.023961410 & 3.274569672 \\ \mathrm{C} & -2.910286518 & 4.120538364 & 3.393650867 \\ \mathrm{~N} & -1.636219165 & 3.821486535 & 3.464038249 \\ \mathrm{C} & -1.613703435 & 2.435040863 & 3.386202281 \\ \mathrm{C} & -0.561447610 & 1.504272179 & 3.405184789 \\ \mathrm{~N} & 0.725351774 & 1.842244698 & 3.512920206 \\ \mathrm{~N} & -0.881322861 & 0.195222532 & 3.308262927 \\ \mathrm{C} & -2.170912367 & -0.142317106 & 3.200360654 \\ \mathrm{~N} & -3.248727141 & 0.638200305 & 3.171019816 \\ \mathrm{C} & -2.897057021 & 1.930893592 & 3.269189022 \\ \mathrm{H} & -3.308874376 & 5.120529864 & 3.423529284 \\ \mathrm{H} & 1.446750664 & 1.138526602 & 3.372583166 \\ \mathrm{H} & 0.964571315 & 2.817331740 & 3.492813714 \\ \mathrm{H} & -2.349690413 & -1.208917364 & 3.135043748 \\ \mathrm{H} & 1.128520864 & -5.057994322 & 3.526271130 \\ \mathrm{~N} & 1.471520484 & -4.117112320 & 3.430975165 \\ \mathrm{C} & 0.530987859 & -3.112083159 & 3.445933998 \\ \mathrm{O} & -0.664608919 & -3.322148874 & 3.564886675 \\ \mathrm{~N} & 1.045615710 & -1.845951068 & 3.315750653 \\ \mathrm{C} & 2.374600620 & -1.494494488 & 3.175602943\end{array}$




$\begin{array}{rrrr}\mathrm{O} & 2.687293341 & -0.311875778 & 3.068737376 \\ \mathrm{C} & 3.306222775 & -2.599505571 & 3.168142034 \\ \mathrm{C} & 4.767653400 & -2.316663570 & 3.019983565 \\ \mathrm{C} & 2.813786719 & -3.838612203 & 3.294563886 \\ \mathrm{H} & 0.333241109 & -1.079714749 & 3.309640681 \\ \mathrm{H} & 5.342116367 & -3.239924428 & 3.031632064 \\ \mathrm{H} & 4.961610277 & -1.790621716 & 2.087314975 \\ \mathrm{H} & 5.116336808 & -1.673423231 & 3.826518591 \\ \mathrm{H} & 3.461793964 & -4.704110251 & 3.295882965 \\ \mathrm{H} & -2.128066321 & -5.028353720 & 0.189752088 \\ \mathrm{~N} & -1.301309542 & -4.459882570 & 0.104741610 \\ \mathrm{C} & -1.480471984 & -3.074896608 & 0.116561318 \\ \mathrm{O} & -2.628599142 & -2.619608496 & 0.228162234 \\ \mathrm{~N} & -0.400765943 & -2.266810879 & 0.005254001 \\ \mathrm{C} & 0.818530505 & -2.797494121 & -0.114125514 \\ \mathrm{~N} & 1.855485191 & -1.962178936 & -0.222289835 \\ \mathrm{C} & 1.029419481 & -4.205654011 & -0.129010520 \\ \mathrm{C} & -0.048191616 & -4.992751158 & -0.018004191 \\ \mathrm{H} & 0.013391540 & -6.071077171 & -0.016415412 \\ \mathrm{H} & 2.015598741 & -4.631049672 & -0.223572668 \\ \mathrm{H} & 2.786192442 & -2.335025439 & -0.261296225 \\ \mathrm{H} & 1.734770252 & -0.943994927 & -0.108170483\end{array}$

Tetramer 9 - GC-CG

$\begin{array}{lrrr}\mathrm{H} & -2.135188422 & 5.035260905 & -0.146754760 \\ \mathrm{~N} & -1.307305565 & 4.469429934 & -0.065592656 \\ \mathrm{C} & -0.004042501 & 4.887005860 & 0.066319186 \\ \mathrm{~N} & 0.844146002 & 3.898278929 & 0.146651742 \\ \mathrm{C} & 0.055273397 & 2.758293790 & 0.062920655 \\ \mathrm{C} & 0.413973979 & 1.387095838 & 0.092721127 \\ \mathrm{O} & 1.541110686 & 0.887778524 & 0.203039132 \\ \mathrm{~N} & -0.700007225 & 0.562472505 & -0.022079726 \\ \mathrm{C} & -1.994985640 & 1.001942901 & -0.149412665 \\ \mathrm{~N} & -2.935030350 & 0.049200089 & -0.248418098 \\ \mathrm{~N} & -2.343314147 & 2.277188808 & -0.177592940 \\ \mathrm{C} & -1.275967980 & 3.095580734 & -0.068479170 \\ \mathrm{H} & -0.549988506 & -0.456845579 & 0.006521469 \\ \mathrm{H} & 0.253878274 & 5.932308498 & 0.097644373 \\ \mathrm{H} & -2.757142852 & -0.928740518 & -0.017868306 \\ \mathrm{H} & -3.880460843 & 0.385958898 & -0.217698577 \\ \mathrm{H} & -4.755254235 & 3.036679869 & 3.204870870 \\ \mathrm{~N} & -3.751134604 & 3.062954369 & 3.275343681 \\ \mathrm{C} & -3.081792054 & 1.837466600 & 3.251151143 \\ \mathrm{O} & -3.744199337 & 0.794254282 & 3.146927104 \\ \mathrm{~N} & -1.731827585 & 1.818651658 & 3.342796825 \\ \mathrm{C} & -1.056085852 & 2.964680546 & 3.454827613 \\ \mathrm{~N} & 0.275270935 & 2.898707271 & 3.543499106\end{array}$




$\begin{array}{rrrr}\text { C } & -1.713353012 & 4.227562984 & 3.481990628 \\ \mathrm{C} & -3.049277101 & 4.230634237 & 3.390407893 \\ \mathrm{H} & -3.633578991 & 5.138981495 & 3.399271291 \\ \mathrm{H} & -1.164585317 & 5.151392368 & 3.570569886 \\ \mathrm{H} & 0.809270014 & 3.747531929 & 3.576569995 \\ \mathrm{H} & 0.774704635 & 2.004474466 & 3.420179723 \\ \mathrm{H} & 1.160901374 & -5.104920031 & 3.525577996 \\ \mathrm{~N} & 1.497256623 & -4.161419579 & 3.431694851 \\ \mathrm{C} & 2.796268240 & -3.734222974 & 3.289087422 \\ \mathrm{~N} & 2.900305987 & -2.436777864 & 3.192804848 \\ \mathrm{C} & 1.592168613 & -1.977824609 & 3.276682855 \\ \mathrm{C} & 1.075603479 & -0.658347481 & 3.233340380 \\ \mathrm{O} & 1.692966336 & 0.407009965 & 3.107635897 \\ \mathrm{~N} & -0.309827620 & -0.645253630 & 3.353948092 \\ \mathrm{C} & -1.098054311 & -1.760719564 & 3.498001667 \\ \mathrm{~N} & -2.418247058 & -1.541911239 & 3.600075305 \\ \mathrm{~N} & -0.629545994 & -2.996520419 & 3.538930929 \\ \mathrm{C} & 0.714529965 & -3.031933606 & 3.424455064 \\ \mathrm{H} & -0.788179579 & 0.266947243 & 3.316505196 \\ \mathrm{H} & 3.619552272 & -4.428239604 & 3.262510784 \\ \mathrm{H} & -2.850591255 & -0.649193943 & 3.360702530 \\ \mathrm{H} & -2.984882921 & -2.370629919 & 3.581686530 \\ \mathrm{H} & -2.128066321 & -5.028353720 & 0.189752088 \\ \mathrm{~N} & -1.301309542 & -4.459882570 & 0.104741610 \\ \mathrm{C} & -1.480471984 & -3.074896608 & 0.116561318 \\ \mathrm{O} & -2.628599142 & -2.619608496 & 0.228162234 \\ \mathrm{~N} & -0.400765943 & -2.266810879 & 0.005254001 \\ \mathrm{C} & 0.818530505 & -2.797494121 & -0.114125514 \\ \mathrm{~N} & 1.855485191 & -1.962178936 & -0.222289835 \\ \mathrm{C} & 1.029419481 & -4.205654011 & -0.129010520 \\ \mathrm{C} & -0.048191616 & -4.992751158 & -0.018004191 \\ \mathrm{H} & 0.013391540 & -6.071077171 & -0.016415412 \\ \mathrm{H} & 2.015598741 & -4.631049672 & -0.223572668 \\ \mathrm{H} & 2.786192442 & -2.335025439 & -0.261296225 \\ \mathrm{H} & 1.734770252 & -0.943994927 & -0.108170483 \\ & & & \end{array}$

Tetramer 10 - TA-AT

$\begin{array}{lrrr}\mathrm{H} & -2.133784727 & 4.978271539 & -0.147869118 \\ \mathrm{~N} & -1.302053587 & 4.418456156 & -0.065291589 \\ \mathrm{C} & -1.471763087 & 3.052592908 & -0.088210437 \\ \mathrm{O} & -2.563447976 & 2.520083584 & -0.199506991 \\ \mathrm{~N} & -0.309584786 & 2.330451166 & 0.024629724 \\ \mathrm{C} & 0.973348734 & 2.826924966 & 0.154857794 \\ \mathrm{O} & 1.922859649 & 2.053710250 & 0.246247206 \\ \mathrm{C} & 1.076973094 & 4.268455278 & 0.171511682 \\ \mathrm{C} & 2.426748286 & 4.898263080 & 0.310000169 \\ \mathrm{C} & -0.051324978 & 4.981767866 & 0.062014586\end{array}$




$\begin{array}{rrrr}\mathrm{H} & -0.434995365 & 1.291747557 & 0.024649401 \\ \mathrm{H} & 2.348222086 & 5.982872728 & 0.306910369 \\ \mathrm{H} & 2.902767131 & 4.580289227 & 1.235477715 \\ \mathrm{H} & 3.078560796 & 4.588922446 & -0.505650383 \\ \mathrm{H} & -0.036295635 & 6.062849394 & 0.068247486 \\ \mathrm{H} & -4.732964538 & 3.004663028 & 3.204568689 \\ \mathrm{~N} & -3.729899019 & 3.023961410 & 3.274569672 \\ \mathrm{C} & -2.910286518 & 4.120538364 & 3.393650867 \\ \mathrm{~N} & -1.636219165 & 3.821486535 & 3.464038249 \\ \mathrm{C} & -1.613703435 & 2.435040863 & 3.386202281 \\ \mathrm{C} & -0.561447610 & 1.504272179 & 3.405184789 \\ \mathrm{~N} & 0.725351774 & 1.842244698 & 3.512920206 \\ \mathrm{~N} & -0.881322861 & 0.195222532 & 3.308262927 \\ \mathrm{C} & -2.170912367 & -0.142317106 & 3.200360654 \\ \mathrm{~N} & -3.248727141 & 0.638200305 & 3.171019816 \\ \mathrm{C} & -2.897057021 & 1.930893592 & 3.269189022 \\ \mathrm{H} & -3.308874376 & 5.120529864 & 3.423529284 \\ \mathrm{H} & 1.446750664 & 1.138526602 & 3.372583166 \\ \mathrm{H} & 0.964571315 & 2.817331740 & 3.492813714 \\ \mathrm{H} & -2.349690413 & -1.208917364 & 3.135043748 \\ \mathrm{H} & 1.128520864 & -5.057994322 & 3.526271130 \\ \mathrm{~N} & 1.471520484 & -4.117112320 & 3.430975165 \\ \mathrm{C} & 0.530987859 & -3.112083159 & 3.445933998 \\ \mathrm{O} & -0.664608919 & -3.322148874 & 3.564886675 \\ \mathrm{~N} & 1.045615710 & -1.845951068 & 3.315750653 \\ \mathrm{C} & 2.374600620 & -1.494494488 & 3.175602943 \\ \mathrm{O} & 2.687293341 & -0.311875778 & 3.068737376 \\ \mathrm{C} & 3.306222775 & -2.599505571 & 3.168142034 \\ \mathrm{C} & -1.767653400 & -2.316663570 & 3.019983565 \\ \mathrm{C} & -2.319934398 & -2.202293798 & 0.196531257 \\ \mathrm{C} & 2.813786719 & -3.838612203 & 3.294563886 \\ \mathrm{H} & 0.333241109 & -1.079714749 & 3.309640681 \\ \mathrm{H} & 5.342116367 & -3.239924428 & 3.031632064 \\ \mathrm{H} & 4.961610277 & -1.790621716 & 2.087314975 \\ \mathrm{H} & 5.116336808 & -1.673423231 & 3.826518591 \\ \mathrm{H} & 3.461793964 & -4.704110251 & 3.295882965 \\ \mathrm{H} & -2.128866628 & -4.989349217 & 0.189661216 \\ \mathrm{~N} & -1.307060099 & -4.415850925 & 0.105122834 \\ \mathrm{C} & -0.000570752 & -4.822011602 & -0.023504880 \\ \mathrm{~N} & 0.853169241 & -3.831687049 & -0.113055448 \\ \mathrm{C} & 0.056811853 & -2.696319660 & -0.038538322 \\ & 1.598475557 & -0.842762277 & -0.200532306 \\ \mathrm{~N} & -0.667345165 & -0.453344592 & 0.023923316 \\ \end{array}$




\begin{tabular}{rrrr}
$\mathrm{H}$ & 0.264878032 & -5.865469379 & -0.045432307 \\
$\mathrm{H}$ & 1.769488951 & 0.152033730 & -0.072215031 \\
$\mathrm{H}$ & 2.365605264 & -1.490517200 & -0.181808744 \\
$\mathrm{H}$ & -2.678803598 & -0.179360342 & 0.215455561 \\
\hline
\end{tabular}




\section{References}

- Complete list of authors of Reference (11):

Olson, W. K.; Bansal, M.; Burley, S. K.; Dickerson, R. E.; Gerstein, M.; Harvey, S. C.; Heinemann, U.; Lu, X.-J.; Neidle, S,; Shakked, Z.; Sklenar, H.; Suzuki, M.; Tung, C. H.; Westhof, E.; Wolberger, C.; Berman, H. M. J. Mol. Biol. 2001, 313, 229.

- Complete list of authors of Reference (13):

MOLPRO, version 2006.3, a package of ab initio programs, Werner, H.-J.; Knowles, P. J.; Lindh, R.; Manby, F. R.; Schuetz, M.; Celani, P.; Korona, T.; Rauhut, G.; Amos, R. D.; Bernhardsson, A.; Berning, A.; Cooper, D. L.; Deegan, M. J. O.; Dobbyn, A. J.; Eckert, F.; Hampel, C.; Hetzer, G.; Lloyd, A. W.; McNicholas, S. J.; Meyer, W.; Mura, M. E.; Nicklass, A.; Palmieri, P.; Pitzer, R.; Schumann, U.; Stoll, H.; Stone, A. J.; Tarroni, R.; Thorsteinsson, T. see http://www.molpro.net; Cardiff, UK, 2006. 